Revue d'histoire du XIXe siècle

Société d'histoire de la révolution de 1848 et des

révolutions du XIXe siècle

$23 \mid 2001$

Nouvelles approches en histoire économique

\title{
Simone Delattre, Les Douze heures noires. La nuit à Paris au XIX ${ }^{e}$ siècle
}

Paris, Albin Michel, 2000, 679 p.

Jacques Rougerie

\section{(2) OpenEdition \\ Journals}

Édition électronique

URL : http://journals.openedition.org/rh19/328

DOI : $10.4000 /$ rh 19.328

ISSN : $1777-5329$

Éditeur

La Société de 1848

Édition imprimée

Date de publication : 1 décembre 2001

Pagination : 253-258

ISSN : 1265-1354

Référence électronique

Jacques Rougerie, "Simone Delattre, Les Douze heures noires. La nuit à Paris au XIXe siècle », Revue d'histoire du XIXe siècle [En ligne], 23 | 2001, mis en ligne le 28 juin 2005, consulté le 10 décembre 2020. URL : http://journals.openedition.org/rh19/328 ; DOI : https://doi.org/10.4000/rh19.328

Ce document a été généré automatiquement le 10 décembre 2020.

Tous droits réservés 


\section{Simone Delattre, Les Douze heures noires. La nuit à Paris au XIX $X^{e}$ siècle}

Paris, Albin Michel, 2000, 679 p.

Jacques Rougerie

1 Voici un livre d'une qualité rare, et qui assurément fera date. Paris révélé par sa nuit. La manière est inattendue d'écrire son histoire; elle eut assurément plu à Hugo : c'est à lui d'ailleurs qu'est emprunté le titre, comme en forme de dédicace.

2 Le projet était audacieux ; la réussite est remarquable. On nous offre une histoire d'une étonnante richesse, qui porte le regard (la lumière ?) dans les directions les plus variées : histoire des faits et des regards sur les faits, du social et des représentations du social, du temps et des façons de le vivre, des lieux de plaisir et de loisirs, des anxiétés bourgeoises, et aussi d'une sensibilité qui s'affine, d'une réceptivité croissante à la nuit, de la sensibilité à l'obscur du "nouvel individu nocturne". Histoire au fond qui est celle des incertitudes de la nouvelle société post-révolutionnaire, entrant dans l'ère ambiguë de la modernité.

Cette histoire très neuve se fonde sur une maîtrise exceptionnelle de l'information, sur une connaissance du Paris du XIX ${ }^{e}$ siècle qu'on prend rarement en défaut, avec une remarquable qualité de présentation synthétique des travaux existants. L'écriture est d'une rare élégance, un rien précieuse, et qu'on envie: c'est exactement celle qui convient à des analyses aussi fines que nuancées. Le livre --que ne saurait épuiser une seule lecture, et surtout pas hâtive--, se savoure. Il est d'une telle densité et d'une telle subtilité qu'on peut parfois s'y perdre, à force de nuances et de nuances dans la nuance, jamais inutiles pourtant. L'auteur a toujours l'inquiétude, ou le remords, d'une explication trop simple. D'où l'ambiguïté souvent de cette histoire, qui pourrait bien être en vérité celle de toute histoire.

4 La nuit, "antidote à la rationalité d'une ville diurne, surface trompeuse", est bien sûr toujours énigmatique. Elle révèle, par opposition au jour "bien pensant et laborieux"; mais elle "révèle et égare à la fois", car "Paris n'est jamais ce que l'on croit". Au cours du premier XIXe siècle, nous nous trouvons à un moment de transition entre la nuit "indomptée" de type ancien, nuit libre, nuit anarchique et du couvre-feu, et la nuit 
parisienne normalisée, humanisée (haussmannisée ?), pour l'essentiel de 1830 à 1860 . On passerait donc d'une culture nocturne à une autre.

Il était bon en tout cas de nous rappeler que l'heure est alors solaire (en réalité, depuis 1811, le temps moyen solaire de Paris). On ne s'étonnera plus désormais de voir l'ouvrier, ou l'insurgé barricadier se lever si tôt matin, vers les trois ou quatre heures : c'est le moment où point le jour. Cependant, si mes comptes sont exacts, au solstice d'été, jour le plus long, le soleil se lève vers quatre heures et non trois, et le jour le plus court s'achève à seize heures et non quinze.

En prologue nous est offerte une remarquable histoire du regard des Parisiens sur la nuit, étayée de textes qui témoignent d'une érudition, plutôt d'une culture rares. Histoire d'un regard en quête de l'inédit, de Rétif et Mercier à Nerval, Baudelaire, Hugo bien sûr, et aussi tant d'autres moins connus. Ce prologue, décrivant le "contexte éditorial où s'insèrent les représentations nocturnes de la grande ville", fait affleurer les problèmes posés. On constate des changements de l'angle du regard, avec la valorisation par la sensibilité romantique du versant nocturne de Paris, de la nuit féminine et de son silence, "entre répulsion ancienne et célébration neuve". D'un autre côté l'espace nocturne n'est plus tout à fait le même. Se trouve soulevé déjà le problème de la représentativité de ces visions, de ces représentations. Est-ce mutation réelle ou mutation de la représentation? Ce prologue est aussi le rappel discret que l'histoire ici faite n'est elle aussi que celle d'un regard : regard sur le regard.

Vient la suite des chapitres, aux titres joliment accrocheurs --qu'on l'entende au meilleur sens du terme. L'histoire de la nuit parisienne se déroule avec une belle logique, du plus clair au plus obscur, de la lumière aux bas-fonds.

"Lumières". C'est la diffusion --dans une histoire très matérielle-- de l'éclairage au gaz qui fait l'unité chronologique de la période. On n'a pas pour autant une banale histoire de l'éclairage, mais une histoire du progrès de la lumière, de la rareté à la profusion et à la superfluité. Lumière "artificielle", qui civilise et assainit, mais désenchante aussi la nuit, car elle n'est jamais qu'un artifice. Peut-être eût-il été juste de rendre ici à Rambuteau, préfet tellement décrié, la paternité essentielle de ce progrès dans l'ancien Paris, de 1837 (202 becs de gaz) à 1847 (8 600). Avec Haussmann, il s'agira surtout de la disparition du réverbère à huile.

9 "Noctambulismes". Dans cette nuit déambulent les "contemplateurs élitistes de la nuit", les noctambules. Apparaît un "nouvel individu nocturne", avec une "façon inédite d'user de son temps", la "volonté d'intimité avec la ville, la sensibilité à l'obscur : bohême jeune, hétéroclite de "citoyens libres et sensibles" qui cherchent le divertissement élégant et canaille : on se donne le frisson aux Halles. La nuit de cette "petite société oligarchique de l'oisiveté" est en effet élitiste : l'homme du peuple qui déambule (ou déambulerait?) dans le Paris nocturne ne peut être vu que comme un "rôdeur de nuit" : le terme apparaît alors. Peuple nocturne, peuple dangereux.

Par une transition peut-être un peu laborieuse --la rencontre supposée, vers les quatre ou cinq heures du matin, de ceux qui vont au travail diurne et de ceux qui reviennent de leurs plaisirs nocturnes--, on passe aux activités des travailleurs de la nuit. "Êtres obscurs, occupés à des industries inconnues", "qui se faufilent dans les rues plus qu'ils ne s'y exhibent". Ce sont là les fonctions nécessaires à la vie de la ville : balayeurs, vidangeurs, chiffonniers, marchands et marchandes des Halles, ce lieu qui ne dort jamais. La description est attrayante et réaliste ; c'est peut-être pourtant un peu trop insister sur l'étrangeté de ces braves travailleurs; un peu fictif aussi, car, somme toute, 
on ne compte jamais que 160 vidangeurs (qui sont des ouvriers très bien payés pour leur besogne sale), tout au plus 2000 chiffonniers sous Juillet, peut-être 4000 ensuite, 250 balayeurs sous la Restauration, puis 800 . Ils ont droit à toutes les attentions ? C'est beaucoup de bruit pour peu de monde. Bien peu de place en regard a été faite aux 2500 puis 4500 ouvriers boulangers : ces "troglodytes" ne sont pas dans la rue, et pour cause ; ne sont-ils pas pourtant le meilleur exemple de la nuit laborieuse?

11 "Contrôles". La nuit, devenue "la nuit de tous les dangers" est de mieux en mieux contrôlée, sans être pour autant jamais pacifiée. Ici encore, ce n'est pas une histoire de la police qu'on nous offre, mais celle du combat, du face à face de l'ordre et du dérèglement, du processus de modernisation de la sécurité à une époque qui se trouve confrontée à la question de la multitude. Histoire de l'obsession sécuritaire, qui somme toute est redevenue la nôtre. Et en même temps, histoire de l"'affirmation progressive d'un droit au repos et au confort privé", des progrès du privé, donc de la protection du privé. C'est le temps de la sacralisation de la propriété et de la sécurité individuelle (faut-il parler pour autant d'une exigence "laïcisée" de sécurité physique ?).

12 Pendant la "vraie" nuit, de onze heures du soir (heure de fermeture des cabarets) à trois ou quatre heures du matin, circulent les patrouilles grises (de policiers en bourgeois) de la Restauration, sous la monarchie de Juillet celles, parfois comiques, d'une Garde nationale de bourgeois froussards. Une paix semble s'instaurer avec la nuit “quadrillée" du Second Empire, l'îlotage de Paris, les rafles. C'est cependant l'échec, dans une large mesure, du projet haussmannien qui n'a fait au fond que repousser le problème aux lisières, au-delà des barrières, dans la banlieue puis bientôt la zone. Malgré la montée de la lumière et le progrès de l'ordre, il y aura désormais un Paris lumineux et réglé et un Paris qui reste ombreux et désordonné.

On en vient aux "Soupçons", à l'histoire concrète de la répression de l'errance nocturne (joliment désignée comme la "nuit capturée") dans un territoire toujours mal maîtrisé. Les lieux, les hommes, les séductions féminines, "les attouchements furtifs", sans oublier les antiphysiques (on dit alors plus banalement les pédérastes) du Palais royal puis des Champs-Élysées. Une constatation forte surtout: "La face nocturne de l'existence parisienne en vient à résumer la difficile intégration politique et sociale du peuple de Paris".

14 Car on tombe enfin dans les "bas-fonds". C'est ici tout particulièrement que le projet est affirmé (est-il pour autant réussi ?) d"'entrelacer patiemment examen des réalités sociales et des représentations". Car ce qui est en question fondamentalement, c'est évidemment le délicat problème des classes dangereuses, sur lesquelles il restait beaucoup à dire. "Nouvel imaginaire" des nuits, "inflexions" du discours sur le crime, mais aussi le crime lui-même.

Un tel livre ne se résume pas, on ne peut que l'appauvrir; et qui oserait s'ériger en censeur d'une œuvre pétrie de tant de talent? J'ai bien ça ou là quelque matière à chicane et en ai déjà fait quelques-unes; elles ne se voudraient pas malveillantes.

16 Ne triche-t-on pas un brin avec les heures? Nous avons là un Paris qui ne dort jamais, et dont la nuit est très extensible. À jouer franchement le jeu, est-ce que cette histoire, d'abord nocturne, ne tend pas à empiéter parfois, souvent, sur le vespéral et le matinal, voire carrément sur le jour. ? Si le balayage se fait à partir de cinq heures le matin en été et sept heures en hiver, ou, après 1853 à trois ou quatre heures, c'est déjà le petit jour. Nous avons de bien jolies pages sur le chiffonnier et sa place dans la littérature. Mais celui-ci est tout autant sinon davantage un travailleur du jour que de la nuit. Sa 
divagation est au mieux vespérale, pas vraiment nocturne --c'est le cas du chiffonnier étudié par Le Play que Simone Delattre connait bien. En 1829, interdiction d'ailleurs avait été faite de chiffonner de minuit à cinq heures du matin ; elle n'a peut-être guère été respectée; on ne voit pas pourtant, dans les arrestations étudiées, que des chiffonniers en aient été particulièrement les victimes. Par ailleurs, l'histoire de la résistance à la discipline horaire (toute diurne) de l'ouvrier est peu dans le sujet, comme le problème de l'heure des repas. Peut-on vraiment avancer que la Saint Lundi était bien tolérée sous l'Ancien régime?

Le livre s'étend longuement sur les loisirs nocturnes de l'ouvrier, leur topographie, voire "la revendication croissante d'un droit au divertissement". Y avait-t-il tant de loisirs nocturnes possibles pour l'homme du peuple? Ils sont, ces loisirs, plutôt diurnes ou vespéraux; débits de boisson, bals et guinguettes, carnaval. Et que sait-on à la vérité de "l'abrutissement désastreux des clients attardés des cabarets" ? Je ferai ici observer que la dernière descente de la Courtille est généralement datée de 1838, et que le Carnaval est sévèrement réglementé depuis sa réapparition en 1799. Peut-on surtout affirmer qu'au cours de cette mutation de la culture de la nuit "le temps du loisir populaire perd sa singularité (et) s'aligne sur les classes moyennes" ? Les lieux du "crime" --ou du moins ce qu'on appelle ainsi-- sont évoqués plus qu'étudiés, et c'est dommage. L'évolution de ces lieux aurait pu être étudiée plus à fond: l'analyse des variations de la localisation du crime pourrait en effet mieux faire apparaître le lien des avancées ou des reculs de celui-ci avec les mouvements de la population.

Broutilles que cela! Il y a tout de même ce problème que je ne parviens toujours pas à considérer comme réglé, celui des "classes dangereuses". Classes dangereuses, et probablement aussi classes nocturnes. Simone Delattre me semble acquiescer au fond aux conclusions trop sombres de Louis Chevalier, qu'elle suit de près (admettant notamment son explication démographique, très discutable, d'un Paris redoutablement confronté à un brusque afflux migratoire). Paris surpeuplé, effervescent; inquiétudes, angoisses quant au crime, quant à la nuit. Sûrement ! "Une littérature de la cité obscure a pris naissance", et "l'anxiété est un poncif de la littérature populaire". Mais Paris "criminel" ? Est-ce représentation, ou, osons le mot, réalité sociale? Simone Delattre reconnaît que la prolifération du thème criminel "n'aurait qu'une autonomie partielle vis-à-vis d'une réalité dont elle est l'écume". Il y aurait donc bien quelque part une réalité criminelle. Mais peut-on avancer sans tellement de nuances que Paris, dans les années 1826-1845, était "un coupe-gorge" ? N’est-il pas excessif de parler du "Paris affolé de Charles X" ou d'affirmer que, sous le régime de Juillet, "tout le monde baigne dans la rumeur criminelle". Est-ce vraiment notre cas aujourd'hui ? Faut-il accepter l'idée de l"'incompatibilité croissante" de deux mondes, monde de l'élite, monde populaire? Des thèses de Louis Chevalier, Barrie M. Ratcliffe avait, me semble-t-il, largement fait justice en $1991^{1}$. Simone Delattre plaide vivement contre l'illusion de l'approche statistique: "une histoire purement quantitative de la détresse populaire exprimée dans la vie nocturne serait purement illusoire". Doit-on complètement négliger, pour raisons d'ailleurs de suspicion très légitimes, les comptes de la justice criminelle, qui sont aussi la représentation d'une réalité en effet déformée. Si l'on en croit ces comptes, et particulièrement ceux des arrestations dans le département de la Seine il n'est pas vrai de dire que la criminalité contre les personnes tendrait à diminuer, tandis que croîtrait la criminalité contre les propriétés. Et si on ne les en croit pas, rien ne prouve le contraire. 

sévissent en 1826, puis 1836-1839. On aurait aimé avoir de celles-ci une histoire explicite, comme avait fait autrefois François Gasnault pour l'affaire de la Tour de Nesle, et non pas allusive. Et être sûr qu'elles agissaient surtout de nuit.

misérable et criminel ! C'est surestimer l'importance sociale d'une littérature à la mode et surtout du très médiocre livre de Frégier. Pourquoi alors cette montée apparente de l'anxiété ? Ne faudrait-il pas préférer aux analyses de L. Chevalier celles de G. Procacci, que Simone Delattre utilise d'ailleurs très finement. Mais entre deux ne faut-il pas choisir plus clairement? On alterne habilement le regard sur le regard et une tentative (au demeurant timide) de regard sur la réalité, tant et si bien qu'on ne sait jamais vraiment où l'on en est.

Il me semble aussi qu'il y a un peu trop de misérabilisme dans la description de la détresse populaire, surtout sous la monarchie de Juillet. Compte tenu de la forte mortalité des hospices qu'il faut écarter, ce n'est pas, tout au long de ce premier XIX ${ }^{\mathrm{e}}$ siècle, un Parisien sur trois qui meurt à l'hôpital, mais seulement un sur quatre, avec une belle régularité du Premier Empire à la Seconde République. Qu'importe, dira-ton ? Mais aussi bien la mort à l'hôpital est-elle preuve de grande ou de totale pauvreté ? Barrie M. Ratcliffe encore ${ }^{2}$ a montré que rien ne permettait de dramatiser leur situation, que l'insertion des migrants dans la ville s'était faite non pas sans difficultés, mais sans misère excessive particulière à cette population largement majoritaire dans Paris. À $70 \%$, le Parisien est un immigré.

Toute au souci de "croiser" réalités si difficiles à atteindre et représentations probablement trop fortes de ces réalités, Simone Delattre tend à privilégier parfois, souvent, ce qui n'est qu'un scintillement (agréable à lire au demeurant) d'anecdotes à l'appui de l'argumentation --l'auteur les nomme joliment "l'écume quotidienne des incidents"-- sur ce qui risquerait d'être une analyse trop classiquement "sociale".

Modification de la sensibilité collective, nuit en voie de civilisation, Simone Delattre a voulu courir tous les risques d'une histoire de la sensibilité. Comment faire autrement que littérairement --et dans ce cas, on ne saurait généraliser-- une histoire de la sensibilité à l'obscur ? Comment exprimer le "silence de la nuit" sans être tant soit peu impressionniste ? Est-il si vrai qu'il y a un "nouvel individu nocturne" ailleurs que dans la littérature? Cela nous vaut de belles pages, un peu aventureuses à mon gré : c'est un risque que l'auteur n'a pas craint d'affronter, car elle sait toujours nuancer.

La nuit comme révélateur social, assurément, mais point trop. N'est-il pas un peu outré encore que de dire que "C'est peut-être après minuit que la revendication populaire du droit à la rue, indissociable du grondement insurrectionnel, prend sa forme la plus lancinante". Mais voici que j'ai, tout d'un coup, achevant ce texte, l'impression de n'avoir outrancièrement retenu que quelques propos parfois excessifs, alors que dans le livre de Simone Delattre tout est nuance. C'est que, historien d'un social rétrograde, j'en conviens, j'avoue quant à moi préférer faire une histoire du Paris diurne. Je n'en suis peut-être que mieux placé pour admirer ce que je puis bien appeler l'exploit historique qu'est ce beau livre, et reconnaitre n'avoir pas su suffisamment le dire. 


\section{NOTES}

1.. Barrie M. RATCLIFFE, “Classes laborieuses et classes dangereuses à Paris pendant la première moitié du XIX ${ }^{\mathrm{e}}$ siècle ? The Chevalier Thesis Reexamined", dans French Historical Studies, $\mathrm{n}^{\circ} 17,1991$, pp. 542-574.

2.. Christine PIETTE et Barrie M. RATCLIFFE, "Les migrants et la ville : un nouveau regard sur le Paris de la première moitié du XIX ${ }^{\mathrm{e}}$ siècle", dans Annales de démographie historique, 1993, pp. 263-302. 